
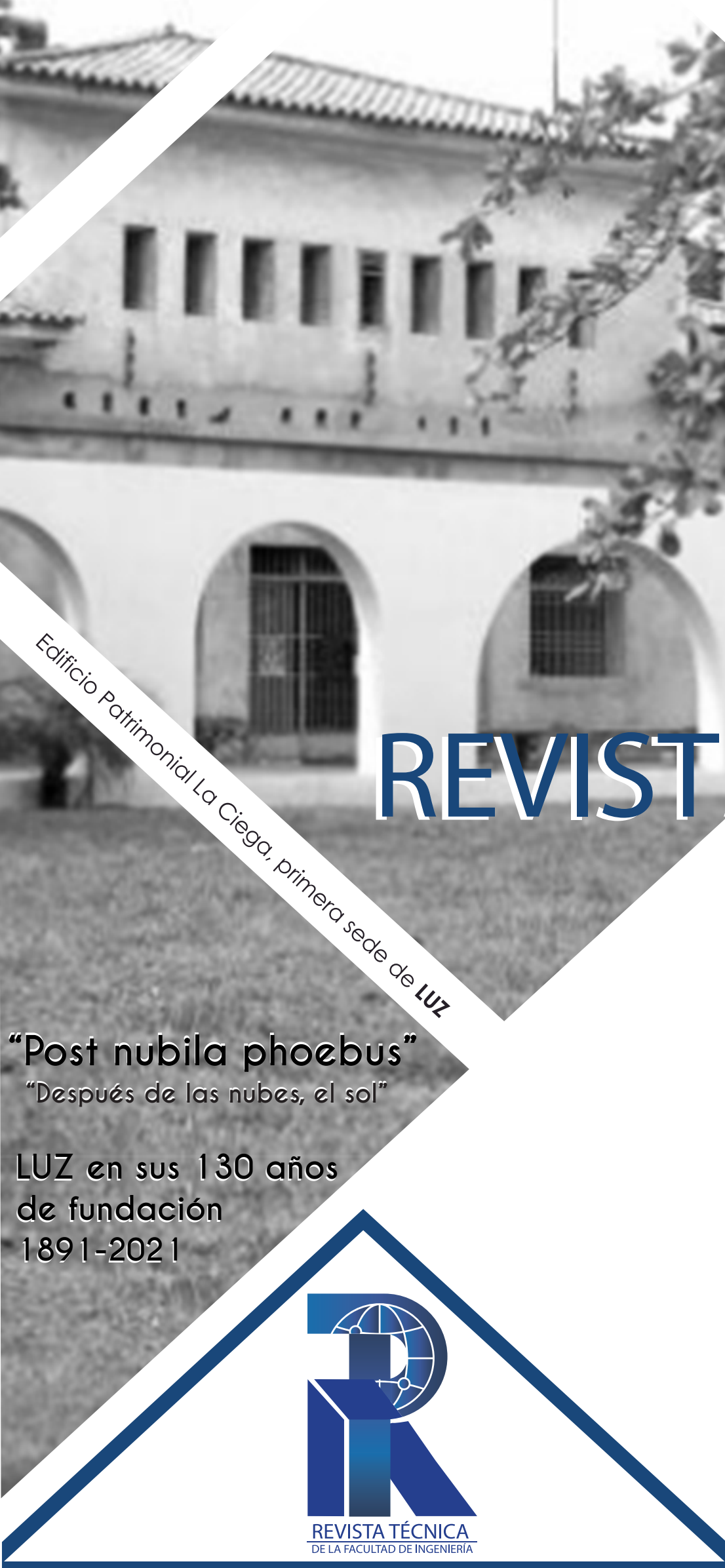

, $18,4,2$,
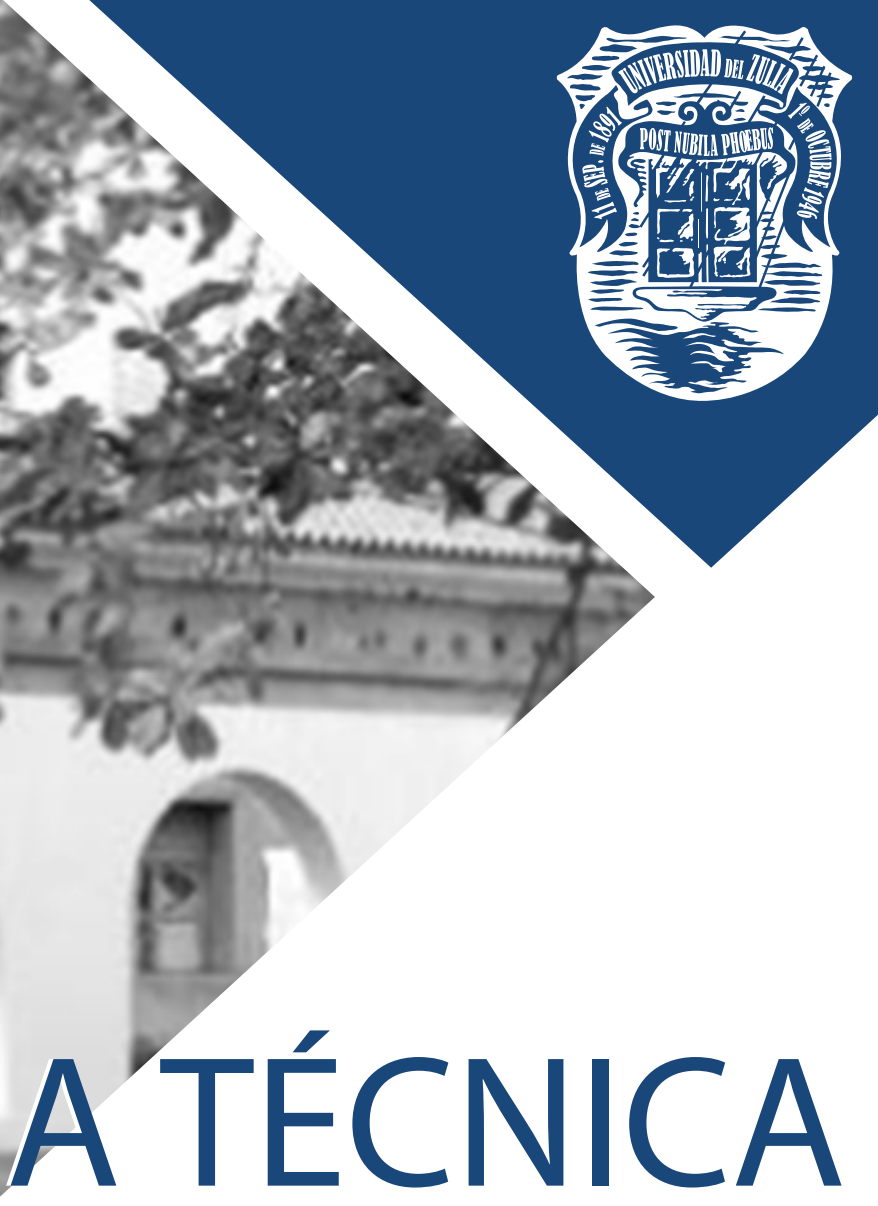
DE LA FACULTAD DE INGENIERÍA

Una Revista Internacional Arbitrada que está indizada en las publicaciones de referencia y comentarios:

- SCOPUS

- SCIELO

- LATINDEX

- DOAJ

- MIAR

- REDIB

- AEROSPACE DATABASE

- CIVIL ENGINEERING ABTRACTS

- METADEX

- COMMUNICATION ABSTRACTS

- ZENTRALBLATT MATH, ZBMATH

- ACTUALIDAD IBEROAMERICANA

- BIBLAT

- PERIODICA

- REVENCYT

UNIVERSIDAD DEL ZULIA 


\title{
Vínculo de la Composición de Recubrimientos SAW con la Composición de Fundentes Obtenidos con Escoria de Acería y Cenizas de Cascarilla del Arroz
}

\author{
R. Najarro ${ }^{1}$, A. Cruz-Crespo ${ }^{2 *}$ (D) L. Perdomo $^{2}$ (D) A. Duffus ${ }^{2}$ (D) G. \\ Almeida $^{2}$ (D) M. J. Morales ${ }^{1}(\mathbb{D})$ \\ ${ }^{1}$ Universidad Técnica de Cotopaxi-La Maná, Av. Los Almendros y calle Pujili sector La \\ Virgen, La Maná - Ecuador. \\ ${ }^{2}$ Centro de Investigaciones de Soldadura, Facultad de Ingeniería Mecánica e Industrial, \\ Universidad Central "Marta Abreu" de Las Villas, CP 54 830, Santa Clara, Cuba. \\ *Autor de correspondencia: acruz@uclv.edu.cu \\ https://doi.org/10.22209/rt.v44n2a06
}

Recepción: 19 de julio de 2020 | Aceptación: 30 de marzo de 2021 | Publicación: 30 de abril 2021

\section{Resumen}

El recargue por soldadura con arco sumergido (SAW, según sus siglas en inglés) se aplica con frecuencia en piezas que trabajan en condiciones de desgaste abrasivo. Los fundentes comerciales que se utilizan son obtenidos a partir de minerales naturales. El objetivo del presente trabajo fue establecer los vínculos entre la composición química de la aleación depositada por SAW y la composición del fundente, elaborado a base de escoria de afino del acero con adición de cenizas de cascarilla del arroz. Se elaboraron fundentes por peletización con base en un diseño de tipo McLean-Anderson. Con los fundentes experimentales se obtuvieron depósitos, que fueron caracterizados químicamente. Se obtuvieron modelos de la composición de los elementos en el metal depositado en función de la composición del fundente y fue realizado un proceso de optimización. Se concluye, que los contenidos de los elementos en el metal depositado muestran una dependencia cuadrática, siendo el contenido de carbono (C) gobernado por el de grafito en el fundente, mientras los contenidos de cromo (Cr), manganeso (Mn) y silicio ( $\mathrm{Si}$ ) son gobernados por el contenido de FeCrMn.

Palabras clave: desgaste abrasivo; escorias de acería; fundente para SAW; recargue duro.

\section{Relationship of the SAW Coatings Composition with the Composition of Fluxes Obtained with Steel Slag and Rice Husk Ashes}

\begin{abstract}
Submerged arc welding (SAW) surfacing is frequently applied on parts that work in abrasive wear conditions. The commercial fluxes are obtained from natural minerals. The objective of this work is to establish the relationship between the chemical composition of the deposited alloy by SAW and the composition of the flux, obtained from steel refining slag with the addition of rice husk ashes. Fluxes were obtained by pelletizing, based on a McLean-Anderson type design. With the experimental fluxes deposits were obtained, which were chemically characterized. Empirical models of the elements composition in the deposited metal as funsion of the flux composition were obtained and an optimization process was carried out. It is concluded that the contents of the elements in the deposited metal show a quadratic dependence, the carbon content $(\mathrm{C})$ is ruled by graphite composition in the flux, while the chromium $(\mathrm{Cr})$, manganese $(\mathrm{Mn})$ and silicon $(\mathrm{Si})$ contents are ruled by the FeCrMn content.
\end{abstract}

Keywords: abrasive wear; hardfacing; SAW fluxes; steel slags. 


\section{Introducción}

El recargue por soldadura con arco sumergido (SAW, según sus siglas en inglés) es de frecuente aplicación en el enfrentamiento al desgaste, ya que brinda una amplia gama de posibilidades en la variación de la composición de los depósitos y una alta productividad del proceso de deposición (Tusek y Suban, 2003; Gulenç y Kahraman, 2003; Shan-Ping et al., 2004; Mendez et al., 2014).

La casi totalidad de los fundentes para proceso SAW son fabricados a partir de minerales naturales. No obstante, se reporta el empleo, como materia prima, de escorias de soldadura de aceros por SAW (Singh y Pandey et al., 2009). En el citado trabajo, se emplea escorias de soldadura de aceros del propio proceso SAW, para la obtención de un nuevo fundente aglomerado, destinado también para la soldadura de unión de estructuras de acero. En publicaciones precedentes de algunos de los autores del presente trabajo, fue utilizado como uno de los componentes de la matriz de un fundente para recargue por $\mathrm{SAW}$, escorias del sistema $\mathrm{SiO}_{2}-\mathrm{MnO}_{\text {, }}$ resultantes de la soldadura por SAW de estructuras de acero y de escorias del sistema $\mathrm{Al}_{2} \mathrm{O}_{3}-\mathrm{MgO}-\mathrm{SiO}_{2}$ de la obtención de ferrocromo y ferrocromomanganeso (Cruz-Crespo et al., 2005; Cruz-Crespo et al., 2017; PerdomoGonzález et al., 2017; Cruz-Crespo et al., 2019).

Por otra parte, las escorias de obtención de acero se caracterizan por la presencia mayoritaria del sistema cuaternario $\mathrm{CaO}-\mathrm{SiO}_{2}-\mathrm{MgO}-\mathrm{Al}_{2} \mathrm{O}_{3}$. El mayor uso de estas escorias ha estado enfocado a aplicaciones de la construcción civil. Las escorias del periodo de oxidación durante la elaboración del acero, por sus relativamente altos contenidos de $\mathrm{Fe}_{2} \mathrm{O}_{3}$ y de $\mathrm{P}_{2} \mathrm{O}_{5}$, no son viables para la elaboración de fundentes. Sin embargo, las escorias del proceso de afino del acero son adecuadas como materia prima para la elaboración de fundentes para SAW, ya que prácticamente no contienen $\mathrm{Fe}_{2} \mathrm{O}_{3}$ y $\mathrm{P}_{2} \mathrm{O}_{5}$ (Najarro-Quintero et al., 2018a).

La cascarilla del arroz constituye un residual agroindustrial al que no se ha encontrado tratamiento sistemático, pese a que muchos autores reportan las potencialidades de sus cenizas para su empleo en el desarrollo de nuevos materiales, sobre todo por sus propiedades pusolánicas (Jarre et al., 2021).

En estudios precedentes de los autores del presente trabajo, fue corroborado el empleo de las escorias de afino del acero como componente de la matriz de un fundente para recargue por SAW, validándose también la adición de cenizas de cascarilla del arroz como aportador de $\mathrm{SiO}_{2}$ al sistema matricial (Najarro-Quintero et al., 2018a; Najarro-Quintero et al., 2018b). El presente trabajo, da continuidad a los referidos estudios, al plantearse como objetivo, establecer los vínculos entre las variables de composición del fundente y la composición química de la aleación depositada por SAW, con base en un diseño experimental de mezclas para una región restringida.

\section{Materiales y Métodos}

Para el estudio fue aplicado un diseño de experimentos de tipo McLean-Anderson, considerando variables independientes, la matriz del fundente $\left(\mathrm{X}_{1}\right)$, el grafito $\left(\mathrm{X}_{2}\right)$ y el FeMnCr $\left(\mathrm{X}_{3}\right)$. En el fundente, el grafito y el FeCrMn constituyen el sistema de aleación. La matriz experimental (Tabla 1) fue obtenida de la siguiente forma: De la matriz completa del diseño (12 combinaciones posibles de variables), luego de aplicar la condición de normalidad $\sum \mathrm{Xi}=100 \%$ y eliminar los puntos con variables fuera de rango; así como al considerar los puntos experimentales coincidentes, solo quedaron 4 experimentos válidos (a, b, c, d). Entre estos puntos fueron añadidos 4 nuevos (ab, bc, cd, da) y uno en el centro (abcd).

Para la obtención de cada fundente experimental, primeramente, se conformó la mezcla de componentes de la matriz. La composición de la matriz del fundente fue establecida en un estudio precedente (NajarroQuintero et al., 2018a), y su relación porcentual de componentes es: 72,99 \% de escoria del horno cuchara de ACINOX Las Tunas, 20,44 \% de cenizas de la combustión de cascarilla de arroz y 6,57 \% de fluorita. Los componentes de la matriz fueron llevados a una granulometría inferior a 0,25 mm; mientras que el FeCrMn y el grafito fueron molidos y tamizados en un rango entre 0,1 y $0,25 \mathrm{~mm}$. Las composiciones de las materias primas utilizadas para la conformación de los fundentes experimentales, se muestran en la Tabla 2. 
Tabla 1. Matriz experimental y composición de los fundentes aglomerados, \% en masa.

\begin{tabular}{cccccccc}
\hline \multicolumn{4}{c}{ Matriz experimental } & \multicolumn{4}{c}{ Fundente aglomerado } \\
\hline Experimento & $\mathbf{X}_{\mathbf{1}}$ & $\mathbf{X}_{\mathbf{2}}$ & $\mathbf{X}_{\mathbf{3}}$ & Matriz & Grafito & FeCrMn & $\mathbf{S i O}_{\mathbf{2}}+\mathbf{N a}_{\mathbf{2}} \mathbf{O}$ \\
\hline a & 88 & 7,0 & 5,0 & 78,68 & 6,26 & 4,47 & 10,59 \\
b & 88 & 2,0 & 10,0 & 78,68 & 1,79 & 8,94 & 10,59 \\
c & 78 & 2,0 & 20,0 & 69,74 & 1,79 & 17,88 & 10,59 \\
d & 78 & 7,0 & 15,0 & 69,74 & 6,26 & 13,41 & 10,59 \\
ab & 88 & 4,5 & 7,5 & 78,68 & 4,02 & 6,71 & 10,59 \\
bc & 83 & 2,0 & 15,0 & 74,21 & 1,79 & 13,41 & 10,59 \\
cd & 78 & 4,5 & 17,5 & 69,74 & 4,02 & 15,65 & 10,59 \\
da & 83 & 7,0 & 10,0 & 74,21 & 6,26 & 8,94 & 10,59 \\
acbd & 83 & 4,5 & 12,5 & 74,21 & 4,02 & 11,18 & 10,59 \\
\hline
\end{tabular}

Tabla 2. Composición química, en \% masa, de componentes de carga de los fundentes.

\begin{tabular}{ccccccccc}
\hline \multicolumn{7}{c}{ Componentes de la matriz del fundente } \\
& $\mathbf{S i O}_{2}$ & $\mathbf{C a O}$ & $\mathbf{C a F}_{2}$ & $\mathbf{C O}_{2}$ & $\mathbf{S O}_{3}$ & $\mathbf{P}_{2} \mathbf{O}_{5}$ & Otros \\
\hline Ceniza, \% & $90-97$ & $*$ & - & - & - & - & $*$ \\
Fluorita, \% & 2,87 & 1,12 & 95,00 & 0,88 & 0,10 & 0,03 & - \\
\hline \multicolumn{7}{c}{ Componentes de la carga de aleación del fundente } \\
\hline FeCrMn & $\mathbf{C r}$ & $\mathbf{M n}$ & $\mathbf{C}$ & $\mathbf{S i}$ & $\mathbf{S}$ & $\mathbf{P}$ & $\mathbf{F e}$ \\
Grafito & 19,45 & 59,02 & 0,11 & 2,17 & 0,0036 & 0,17 & balance \\
\hline
\end{tabular}

*Contenidos variables de los óxidos de $\mathrm{Ca}, \mathrm{Mg}, \mathrm{K}, \mathrm{Na}, \mathrm{Al}, \mathrm{Fe}$, etc., en dependencia de las características de los suelos (Cruz, 2009), -: compuesto o elemento no presente en la materia prima.

Las cargas de $1 \mathrm{~kg}$, correspondientes a cada fundente experimental (Tabla 1), se dosificaron por pesaje en una balanza técnica. Para garantizar la homogeneidad de las cargas; estas fueron mezcladas en un tambor giratorio durante $30 \mathrm{~min}$. La aglomeración se realizó por peletizado, utilizando como aglutinante silicato de sodio en una proporción de un $30 \%$ de la masa seca. Los fundentes obtenidos se secaron al aire, luego se tamizaron a una granulometría entre 0,25 y $2,0 \mathrm{~mm}$ y finalmente se calcinaron en un horno mufla a $350{ }^{\circ} \mathrm{C}$ durante $120 \mathrm{~min}$. La composición porcentual de los componentes del fundente, considerando la adición de $30 \%$ de silicato de sodio, se muestra en la Tabla 1 . La adición de $30 \%$ de silicato de sodio $\left(\mathrm{SiO}_{2}-29,39 ; \mathrm{Na}_{2} \mathrm{O}-10,10\right.$ y $\left.\mathrm{H}_{2} \mathrm{O}-60,51 \%\right)$, aporta $11,85 \mathrm{~g}$ de $\left(\mathrm{SiO}_{2}+\mathrm{Na}_{2} \mathrm{O}\right)$ para $100 \mathrm{~g}$ de la masa seca del fundente (matriz + grafito + $\mathrm{FeCrMn}$, ya que el agua es evaporada en el proceso de calcinación, representando $10,59 \%$ el aporte del silicato al fundente (Tabla 1).

Con los fundentes obtenidos se realizaron depósitos sobre chapas de acero AISI 1020, de dimensiones 150x80x8 mm. Fue utilizado alambre electrodo AWS EL12 de $3 \mathrm{~mm}$, con una corriente de 300 A, un voltaje de arco de $35 \mathrm{~V}$, una velocidad de soldadura $43,2 \mathrm{~m} / \mathrm{h}$ y una altura de capa de fundente de $20 \mathrm{~mm}$. El depósito se realizó en tres camadas superpuestas. De cada depósito se extrajo una muestra para análisis químico, mediante cortes transversales en una tronzadora metalográfica. La composición fue determinada por espectroscopía de emisión atómica.

El procesamiento de resultados se realizó con el programa Statgraphics, obteniéndose las ecuaciones de regresión de los contenidos de C, Cr, Mn y Si en el metal depositado, en función de las variables de entrada del diseño experimental. También se realizó un proceso de optimización multivariable, para definir el depósito de mejor composición con vistas al desgaste abrasivo.

Al depósito seleccionado como mejor en cuanto a composición, se le determinó la dureza HV, en un microdurómetro con carga de $1 \mathrm{~kg}$ y un tiempo de identación de $15 \mathrm{seg}$. Fue también preparada una muestra 
metalográfica, con ataque de nital al $2 \%$. La adquisición de imagen se realizó en un microscopio óptico metalográfico.

\section{Resultados y Discusión}

Los resultados experimentales de la composición química media de los elementos fundamentales de los depósitos, se muestran en la Tabla 3. Las Ecuaciones 1, 2, 3 y 4 reflejan el comportamiento de los contenidos de C, Cr, Mn y Si en el metal depositado, en función de las variables de composición del fundente. En todos los casos el comportamiento es cuadrático, con un alto ajuste de los modelos para el C, Cr y Mn: $\mathrm{R}^{2}=96,84 \%$ y $\mathrm{R}^{2}$ ajustada $=91,56 \%(\mathrm{p}=0,0186)$ para el $\mathrm{C}, \mathrm{R}^{2}=99,00 \%$ y $\mathrm{R}^{2}$ ajustada $=97,34 \%$ para el $\mathrm{Cr}(\mathrm{p}=0,0034), \mathrm{R}^{2}=$ $98,33 \%$ y $\mathrm{R}^{2}$ ajustada $=95,56 \%(\mathrm{p}=0,0072)$ para el $\mathrm{Mn}$. En el caso del $\mathrm{Si}$, el modelo no mostró un alto aujuste $\left(\mathrm{R}^{2}=89,63 \%, \mathrm{R}^{2}\right.$ ajustada $=72,35 \%$ y $\left.\mathrm{p}=0,1030\right)$; no obstante, se observa regularidad para una probabilidad de alrededor de $90 \%$, lo que permite evaluar la tendencia de comportamiento.

Tabla 3. Composición de los depósitos experimentales, aporte del sistema fundente-alambre y coeficientes de transferencias de los elementos al metal depositado.

\begin{tabular}{|c|c|c|c|c|c|c|c|c|c|c|c|c|}
\hline \multirow[b]{2}{*}{ Fundente } & \multicolumn{4}{|c|}{$\begin{array}{c}\text { Composición de los depósitos } \\
(\%)\end{array}$} & \multicolumn{4}{|c|}{$\begin{array}{c}\text { Aporte del sistema fundente- } \\
\text { alambre }(\%)\end{array}$} & \multicolumn{4}{|c|}{ Coeficientes de transferencia } \\
\hline & $\mathbf{C}$ & Mn & $\mathrm{Cr}$ & $\mathbf{S i}$ & $\mathbf{C}$ & Mn & $\mathbf{C r}$ & $\mathbf{S i}$ & $\mathbf{K}_{\mathrm{tC}}$ & $\mathbf{K}_{\mathrm{tMn}}$ & $\mathbf{K}_{\mathrm{tCr}}$ & $\mathbf{K}_{\mathrm{tSi}}$ \\
\hline $\mathrm{a}$ & 2,53 & 1,00 & 0,38 & 1,26 & 4,00 & 1,87 & 0,56 & 0,10 & 0,63 & 0,54 & 0,68 & 12,85 \\
\hline $\mathrm{b}$ & 0,65 & 2,87 & 0,95 & 1,43 & 1,17 & 3,56 & 1,11 & 0,16 & 0,55 & 0,81 & 0,85 & 8,93 \\
\hline $\mathrm{c}$ & 0,77 & 4,80 & 1,34 & 2,59 & 1,18 & 6,93 & 2,23 & 0,28 & 0,65 & 0,69 & 0,60 & 9,11 \\
\hline $\mathrm{d}$ & 2,83 & 3,92 & 1,07 & 2,29 & 4,01 & 5,25 & 1,67 & 0,22 & 0,71 & 0,75 & 0,64 & 10,30 \\
\hline$a b$ & 2,01 & 1,87 & 0,73 & 2,36 & 2,59 & 2,71 & 0,83 & 0,13 & 0,78 & 0,69 & 0,87 & 18,28 \\
\hline $\mathrm{bc}$ & 1,00 & 3,71 & 1,11 & 2,11 & 1,17 & 5,25 & 1,67 & 0,22 & 0,85 & 0,71 & 0,66 & 9,49 \\
\hline $\mathrm{cd}$ & 1,73 & 5,05 & 1,40 & 3,06 & 2,59 & 6,09 & 1,95 & 0,25 & 0,67 & 0,83 & 0,72 & 12,08 \\
\hline $\mathrm{da}$ & 2,50 & 2,33 & 0,73 & 2,16 & 4,00 & 3,56 & 1,11 & 0,16 & 0,62 & 0,66 & 0,66 & 13,49 \\
\hline abcd & 2,09 & 3,67 & 1,12 & 2,33 & 2,59 & 4,40 & 1,39 & 0,19 & 0,81 & 0,83 & 0,81 & 12,19 \\
\hline
\end{tabular}

$\mathrm{K}_{\mathrm{tC}}, \mathrm{K}_{\mathrm{tMn}}, \mathrm{K}_{\mathrm{tCr}}, \mathrm{K}_{\mathrm{tS}}$ : coeficientes de transferencia de C, Mn, Cr y Si, respectivamente.

$$
\begin{aligned}
& \mathrm{C}=0,483333^{*} \mathrm{X}_{1}+0,938333 * \mathrm{X}_{2}+0,748333 * \mathrm{X}_{3}+8,46^{*} \mathrm{X}_{1} * \mathrm{X}_{2}+0,99 * \mathrm{X}_{1} * \mathrm{X}_{3}+8,28 * \mathrm{X}_{2} * \mathrm{X}_{3}, \\
& \mathrm{Cr}=0,672222^{*} \mathrm{X}_{1}-3,10278 * \mathrm{X}_{2}+1,34722 * \mathrm{X}_{3}+4,245^{*} \mathrm{X}_{1} * \mathrm{X}_{2}+0,075^{*} \mathrm{X}_{1} * \mathrm{X}_{3}+5,52 * \mathrm{X}_{2} * \mathrm{X}_{3}, \\
& \mathrm{Mn}=1,66167 * \mathrm{X}_{1}-7,95583^{*} \mathrm{X}_{2}+4,88917 * \mathrm{X}_{3}+10,71 * \mathrm{X}_{1} * \mathrm{X}_{2}-0,135 * \mathrm{X}_{1} * \mathrm{X}_{3}+15,3 * \mathrm{X}_{2} * \mathrm{X}_{3}, \\
& \mathrm{Si}=0,898333^{*} \mathrm{X}_{1}-12,7092 * \mathrm{X}_{2}+2,54583 * \mathrm{X}_{3}+22,86^{*} \mathrm{X}_{1} * \mathrm{X}_{2}+0,315^{*} \mathrm{X}_{1} * \mathrm{X}_{3}+21,96 * \mathrm{X}_{2} * \mathrm{X}_{3},
\end{aligned}
$$

Las gráficas de superficies respuestas (Figuras 1, 2, 3 y 4), correspondientes a las ecuaciones de regresión (Ecuaciones 1, 2, 3 y 4), exhiben la tendencia de comportamiento. Se observa, como era esperado, que a medida que aumenta el contenido de grafito en el sistema de aleación del fundente, tiende a aumentar el contenido de $\mathrm{C}$ en el metal depositado. De igual modo, los contenidos de $\mathrm{Cr}$ y $\mathrm{Mn}$ en los depósitos tienden a ser mayores, en la medida que crece el contenido de FeCrMn en el fundente. En el caso del Si, la tendencia al crecimiento está gobernada por la adición de FeCrMn $\left(\mathrm{X}_{3}\right)$, lo cual corresponde a que este componente del sistema de aleación del fundente aporta directamente $\mathrm{Si}$, al tiempo que aporta $\mathrm{Mn}$ que actua como desoxidante, favoreciendo la reducción del $\mathrm{SiO}_{2}$. 


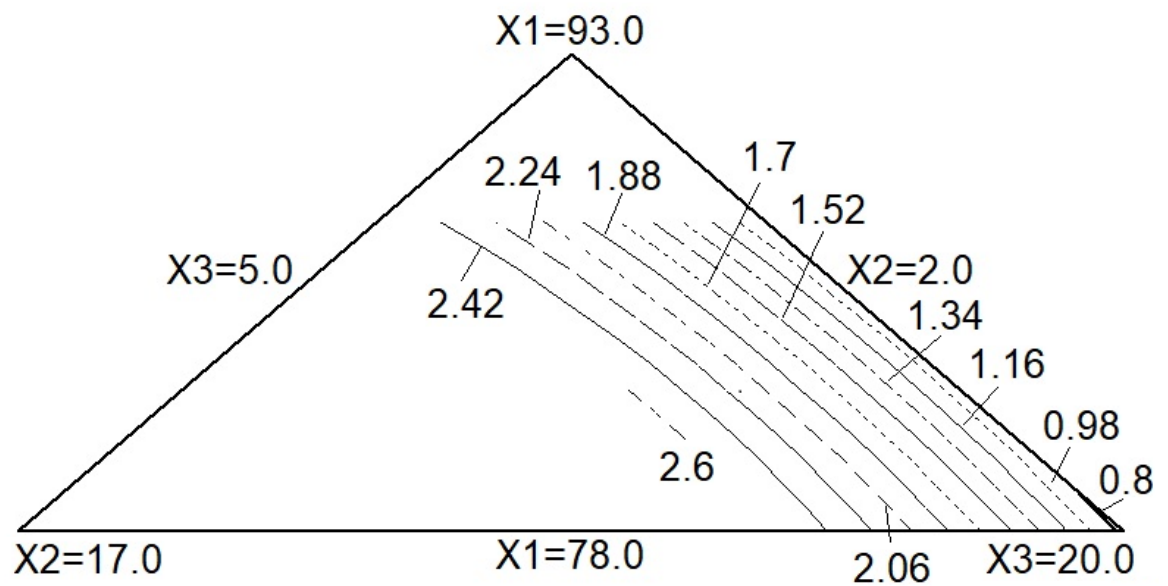

Figura 1. Isolíneas de superficies respuestas del contenido de $\mathrm{C}$ en el depósito (\%), en función de los contenidos de matriz $\left(\mathrm{X}_{1}\right)$, grafito $\left(\mathrm{X}_{2}\right)$ y FeCrMn $\left(\mathrm{X}_{3}\right)$ en el fundente $(\%)$.

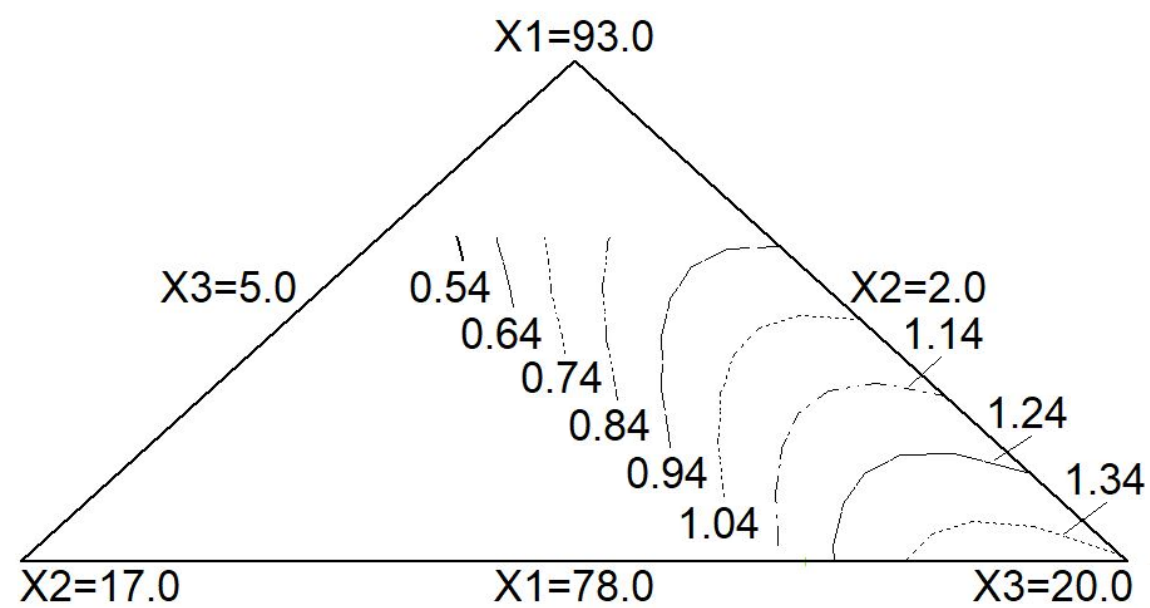

Figura 2. Isolíneas de superficies respuestas del contenido de $\mathrm{Cr}$ en el depósito (\%), en función de los contenidos de matriz $\left(\mathrm{X}_{1}\right)$, grafito $\left(\mathrm{X}_{2}\right)$ y $\mathrm{FeCrMn}\left(\mathrm{X}_{3}\right)$ en el fundente $(\%)$.

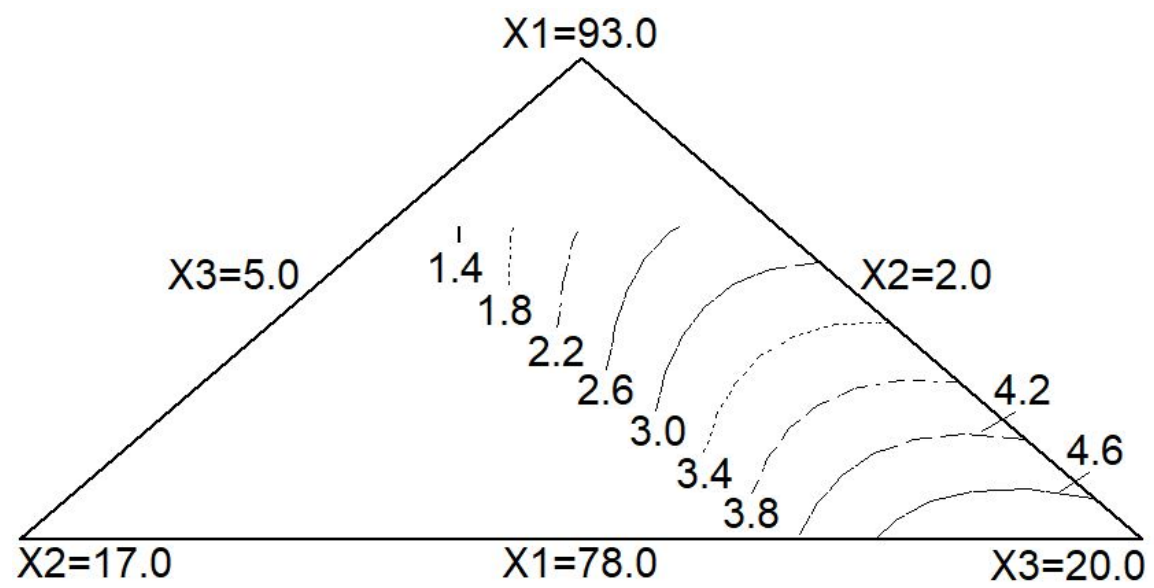

Figura 3. Isolíneas de superficies respuestas del contenido de Mn en el depósito (\%), en función de los contenidos de matriz $\left(\mathrm{X}_{1}\right)$, grafito $\left(\mathrm{X}_{2}\right)$ y FeCrMn $\left(\mathrm{X}_{3}\right)$ en el fundente $(\%)$. 


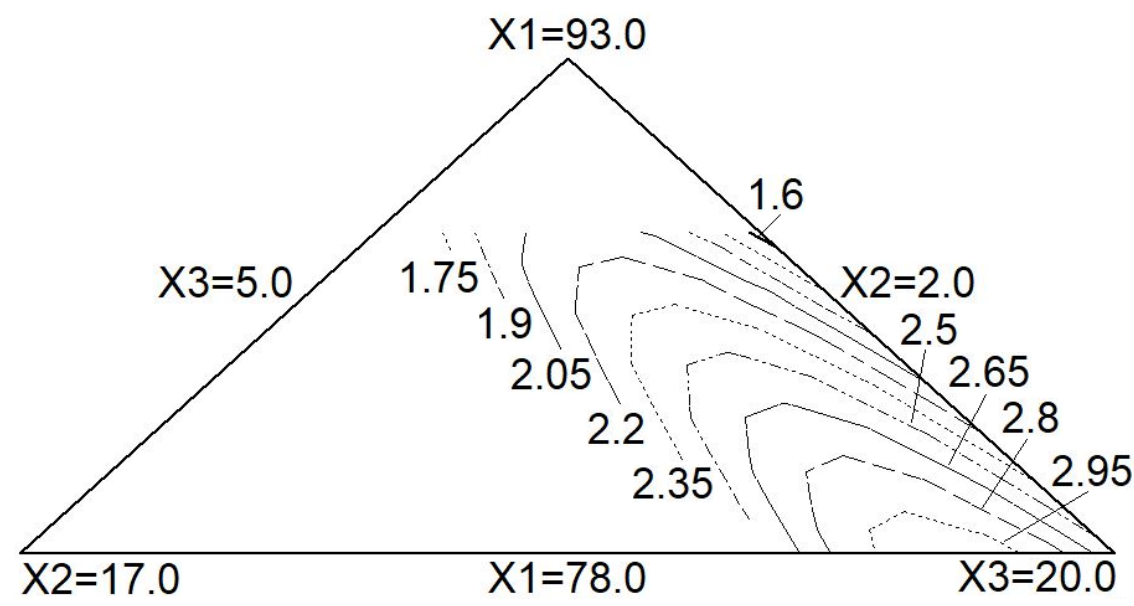

Figura 4. Isolíneas de superficies respuestas del contenido de $\mathrm{Si}$ en el depósito (\%), en función de los contenidos de matriz $\left(\mathrm{X}_{1}\right)$, grafito $\left(\mathrm{X}_{2}\right)$ y FeCrMn $\left(\mathrm{X}_{3}\right)$ en el fundente $(\%)$.

La composición química descrita en la Tabla 3, recalculada al $100 \%$ del sistema ternario Fe-Cr-C, evidencia que en todos los depósitos la cristalización primaria de la posa de fusión ocurre con la formación de austenita (Albertin et al., 2011). A causa de las altas tasas de enfriamiento, propias de un proceso de recargue por soldadura, la austenita se transforma a martensita. El Cr y el Mn, que son formadores de carburos, desplazan las curvas de transformación isotérmica de la austenita hacia la derecha, al tiempo que hacen descender el punto de inicio de la transformación martensítica, provocando la aparición de austenita residual. Dadas la composición química (Tabla 3) y la microestructura resultante del enfriamiento, los depósitos experimentales son relativamente similares a los que aportan consumibles comerciales recomendados para enfrentar el desgaste abrasivo (AWS A 5.13, 2014; Kobe, 2007).

\section{Análisis del proceso de aleación de los depósitos}

En la Tabla 3 se refleja el aporte de elementos de aleación de cada fundente, en combinación con el alambre AWS EL12. Dicho aporte fue determinado con base en la composición química de los componentes de la carga de aleación (Tabla 2) y sus proporciones en la conformación de los fundentes experimentales, condiderando el aporte del silicato de sodio, empleado como aglomerante (Tabla 1). En la determinación del aporte de elementos al depósito por el sistema fundente-alambre, se consideró que la composición del alambre electrodo AWS EL12 es: C-0,09 \%; Mn-0,5\%; Si-0,1 \% (AWS A 5.17, 2001) y que la relación de consumo fundente/alambre es de alrededor de 1,8 (del consumo total $64 \%$ corresponde al fundente y $36 \%$ al alambre).

Al comparar los aportes del sistema fundente-alambre con la composición del metal depositado experimentalmente (Tabla 3 y Figura 5), se observa que existe relativa correspondencia para el $\mathrm{Cr}, \mathrm{Mn}$ y $\mathrm{C}$. El aporte del sistema alambre-fundente difiere de la composición de los depósitos, ya que durante la deposición se verifican procesos de oxidación-reducción. La tendencia a cierta linealidad en el comportamiento entre el aporte del sistema fundente-alambre y lo experimental obtenido en los depósitos (Figura 5), evidencia que las condiciones de concentración gobiernan la aleación del metal, pese a las altas velocidades de enfriamiento que tienen lugar en el recargue por SAW, que pueden conducir al desequilibrio termodinámico del sistema.

Los contenidos de Si en los depósitos experimentales, superan significativamente los valores de este elemento aportados por el sistema fundente-alambre (Tabla 3). Ello se debe a que, en las zonas de altas temperaturas (en la gota y la zona delantera del baño de soldadura) se desarrollan reacciones que provocan la oxidación del metal por la sílice del fundente (Ecuación 5). El [FeO] disuelto en el metal líquido reacciona con el [Mn] y el [C], contenidos en el baño, según las reacciones de las Ecuaciones 6 y 7, liberando al hierro y favoreciendo la reducción del $\mathrm{SiO}_{2}$ en la interfase metal-escoria por la Ecuación 5 (Quintana et al., 2003). El alto contenido de $\mathrm{SiO}_{2}$ en la escoria, aportado por la matriz del fundente (el $\mathrm{SiO}_{2}$ es aportado por la escoria, la ceniza y el silicato), y la presencia de Mn y C que actúan como desoxidantes (Ecuaciones 6 y 7), favorecen la reacción de la Ecuación 5, que conduce al aumento de este elemento en el metal. Es decir:

$$
\begin{gathered}
2 \mathrm{Fe}+\left(\mathrm{SiO}_{2}\right) \rightarrow[\mathrm{Si}]+2[\mathrm{FeO}] \\
{[\mathrm{Mn}]+[\mathrm{FeO}] \rightarrow(\mathrm{MnO})+\mathrm{Fe}} \\
{[\mathrm{C}]+[\mathrm{FeO}] \rightarrow \mathrm{CO}+\mathrm{Fe}}
\end{gathered}
$$


A partir de los contenidos de elementos aportados por el sistema fundente-alambre y la composición de los depósitos obtenidos experimentalmente, se determinaron los coeficientes de transferencias de los elementos $\left(\mathrm{K}_{\mathrm{tE}}=\mathrm{E}_{\text {exp }} / \mathrm{E}_{\text {teórico }} ; \mathrm{K}_{\mathrm{tE}}\right.$ - coeficiente de transferencia del elemento al metal depositado, $\mathrm{E}_{\text {exp }} \mathrm{y} \mathrm{E}_{\text {teórico }}-$ contenidos experimental y teórico del elemento en el metal depositado) (Tabla 3). El coeficiente de transferencia refleja el aprovechamiento efectivo del elemento de aleación, aportado por los consumibles para la conformación del metal depositado. En el caso del C, Mn y Cr, al ser los valores inferiores a 1a unidad, se confirma que hay pérdidas vinculadas a la oxidación de estos elementos. Los valores para el $\mathrm{C}$ y el $\mathrm{Mn}$, en sentido general superan los frecuentemente reportados por la literatura (Potapov, 1989; Quintana et al., 2003); mientras para el Cr se enmarca en el rango reportado, con solo dos valores superiores a dicho rango. En el caso del $\mathrm{Si}$, en correspondencia con lo ya abordado acerca de la reducción del $\mathrm{SiO}_{2}$, el coeficiente de transferencia es significativamente superior a la unidad, evidenciando que ha habido incorporación de este elemento al depósito, luego de un proceso reductivo del óxido desde la escoria, según la Ecuación 4.

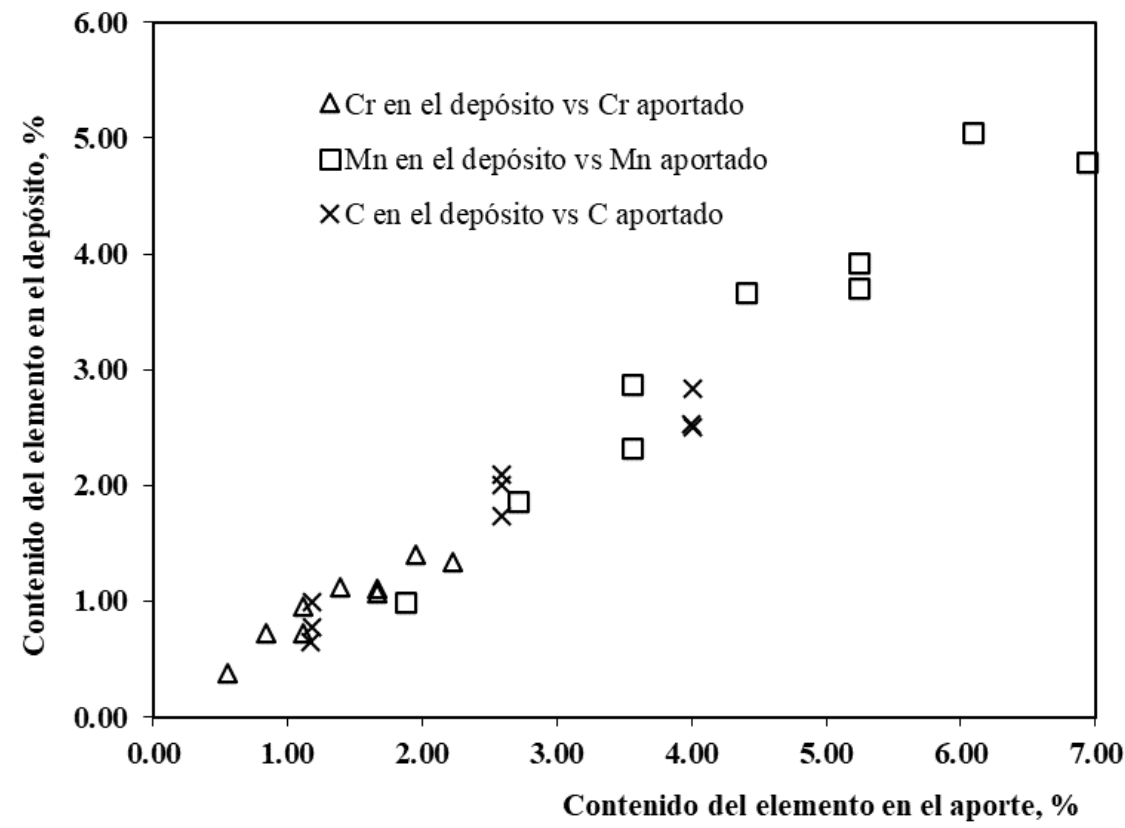

Figura 5. Relación entre los contenidos de los elementos de aleación en el aporte y en el metal depositado.

El comportamiento de tendencia similar de los contenidos de $\mathrm{Cr}$ y $\mathrm{Mn}$ en el metal depositado (Figuras 2 y 3), siendo estos elementos aportados por el mismo componente de carga del fundente (FeCrMn, identificado como la variable $\mathrm{X}_{3}$ en el plan experimental, Tabla 1), es un reflejo de que la aleación del depósito es gobernada por las condiciones de concentración.

En el caso del carbono, debido a la relativa poca cantidad de oxígeno atmosférico y a las altas temperaturas en el arco, su oxidación ocurre predominantemente con los óxidos en el metal líquido (Quintana et al., 2003).

\section{Optimización de la composición del fundente}

Como ya fue planteado, todos los depósitos obtenidos con los fundentes experimentales en combinación con alambre AWS EL12 (Tabla 3), podrían satisfacer los requerimientos para enfrentamiento al desgaste abrasivo. No obstante, desde el punto de vista de la composición del metal depositado, los contenidos de $\mathrm{C}$ y $\mathrm{Cr}$ deben ser los mayores posibles, ya que ello favorece la formación de estructuras duras que limitan la penetración del material abrasivo en la superficie del recubrimiento. La optimización de manera independiente de estas dos respuestas $(\mathrm{C}$ y $\mathrm{Cr}$ en el metal depositado), mediante el procesamiento con el programa Statgraphics, brinda lo siguiente: $\mathrm{C}_{\text {ópt }}=2,7 \%\left(\mathrm{X}_{1}=81,45 ; \mathrm{X}_{2}=7,0\right.$ y $\left.\mathrm{X}_{3}=11,55 \%\right) ; \mathrm{Cr}_{\text {ót }}=1,4 \%\left(\mathrm{X}_{1}=78,0 ; \mathrm{X}_{2}=3,45\right.$ y $\left.\mathrm{X}_{3}=18,55 \%\right)$. Lo anterior evidencia, que los mejores resultados para el contenido de $\mathrm{C}$ y $\mathrm{Cr}$ en el depósito no son coincidentes, en cuanto a composición del fundente, por lo que se requiere encontrar una condición de compromiso, que puede logarse mediante la obtención de la funsión de derseabilidad (Becerra et al., 2014). 
El procesamiento de optimización multivariable se realizó, maximizando el $\mathrm{C}$ y el Cr como respuestas. Se ha obtenido como resultado la gráfica de superficies respuestas de la función "deseada" (Figura 6). Se observa que los mejores resultados tienden hacia el aumento del grafito y la disminución de la matriz para altos valores de FeCrMn, siendo el óptimo de la función deseo igual a 0,82, lo que corresponde a lo siguiente: $\mathrm{C}_{6 \mathrm{pt}}=$ 2,45 y Cr $_{\text {opt }}=1,22 \%\left(\mathrm{X}_{1}=78,0 ; \mathrm{X}_{2}=6,12\right.$ y $\left.\mathrm{X}_{3}=15,88 \%\right)$. Este óptimo es coincidente con el punto experimental "d" (Tabla 1). Por su composición, este depósito podría satisfacer los requerimientos para enfrentamiento al desgaste abrasivo, correspondíendose a la clasificación AWS EFe5, de acuerdo con lo reportado para consumibles de recargue por soldadura (AWS A 5.13, 2010). El Mn en el depósito contribuye a la presencia de austenita residual, lo que favorece para el trabajo en condiciones de abrasión con impacto.

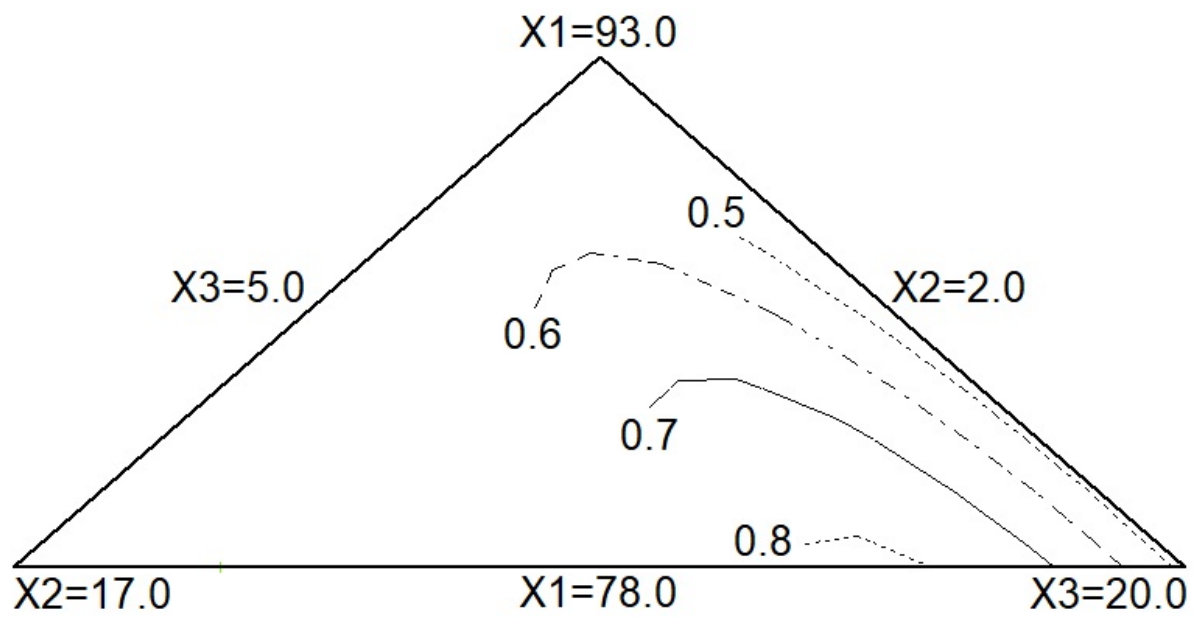

Figura 6. Comportamiento de la función "deseada", en función de los contenidos de matriz $\left(\mathrm{X}_{1}\right)$, grafito $\left(\mathrm{X}_{2}\right)$ y $\mathrm{FeCrMn}\left(\mathrm{X}_{3}\right)$ en el fundente $(\%)$.

La dureza del depósito obtenido con el fundente "d" (549 HV,53 HRc), junto a la microestructura mostrada en la Figura 7, con presencia de martensita en forma de agujas y austenita residual, son también indicios de que el fundente seleccionado como óptimo podría ser adecuado para el enfrentamiento al desgaste abrasivo. Varios autores, entre los cuales se encuentran Gulenc y Kahraman (2003), y Coronado et al. (2009), realizaron estudios que validan el desempeño frente al desgaste abrasivo de recubrimientos de aceros de composición y microestructura similares a la de los depósitos del presente trabajo.

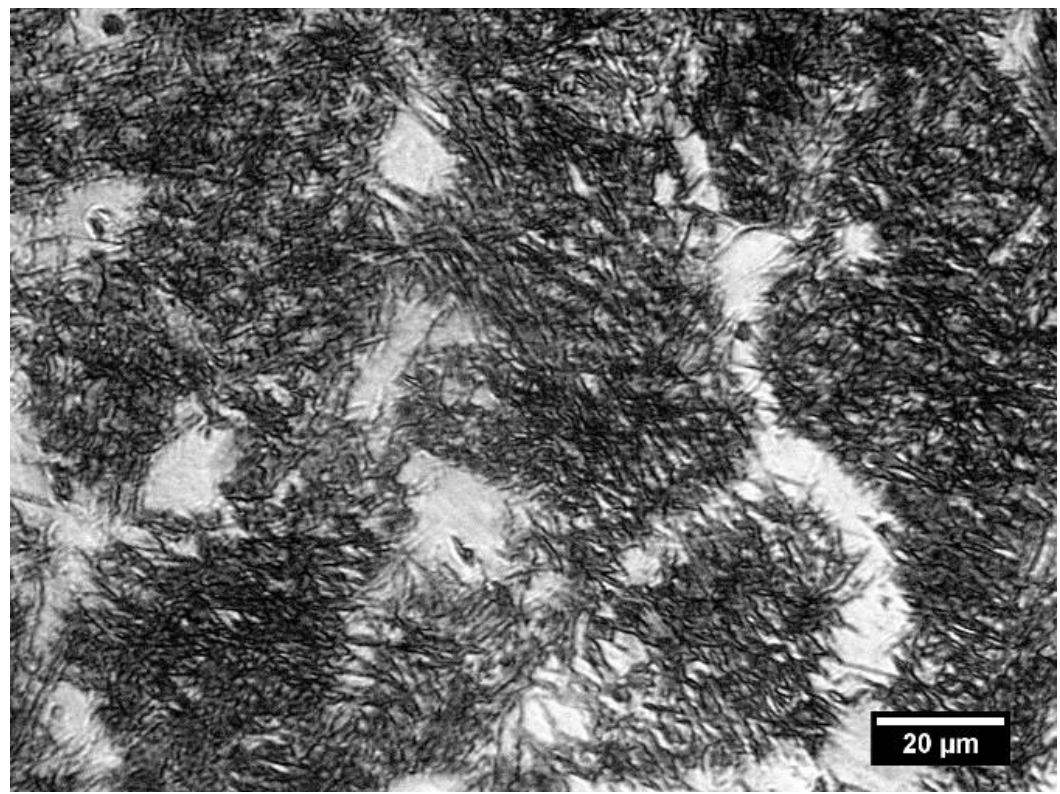

Figura 7. Microestructura del depósito "d" obtenida por microscopía óptica (martencita en forma de agujas-zona oscura y austenita-zona clara). 


\section{Conclusiones}

Se confirma que las escorias del proceso de afino del acero, junto a las cenizas de cascarilla del arroz como aditivo, son viables de emplear como componentes de la matriz de fundentes aglomerados aleados para recargue por proceso SAW.

Los contenidos de C, Cr, Mn y Si en el metal depositado, en función de los contenidos de matriz, grafito y $\mathrm{FeCrMn}$ en los fundentes, muestran una dependencia cuadrática. El contenido de grafito en el fundente gobierna el contenido de $\mathrm{C}$ en los depósitos, mientras los contenidos de $\mathrm{Cr}, \mathrm{Mn}$ y $\mathrm{Si}$ son gobernados por el contenido de FeCrMn en el fundente. Para el C, Cr y Mn la transferencia al depósito desde el aporte del sistema fundente-alambre se manifiesta con pérdidas por los procesos de oxidación de estos elementos, mientras que el contenido de silicio en el metal depositado es superior a lo aportado, a causa de la reducción del $\mathrm{SiO}_{2}$ presente en la escoria.

Las composiciones de los depósitos obtenidos con los fundentes experimentales se enmarcan en rangos similares a las de consumibles comerciales para el enfrentamiento al desgaste abrasivo, caracterizados por el predominio de martencita en la microestructura y la presencia de austenita residual. Del proceso de optimización para maximixar el $\mathrm{C}$ y el $\mathrm{Cr}$, el fundente de mejores propiedades es similar al experimental "d" y se caracteriza por $\mathrm{C}_{\text {ópt }}=2,45$ y Cr $_{\text {opt }}=1,22 \%\left(\mathrm{X}_{1}=78,0 ; \mathrm{X}_{2}=6,12\right.$ y $\left.\mathrm{X}_{3}=15,88 \%\right)$, con dureza en el entorno de $53 \mathrm{HRc}$.

\section{Referencias Bibliográficas}

Albertin, E., Neto, F. B., Teixeira, I. O. (2011). Adequação da composição química e do tratamento térmico de ferros fundidos de alto cromo utilizando termodinâmica computacional. Tecnol. Metal. Mater. Miner., 8(4), 223229.

AWS A 5.13. (2010). Specification for surfacing electrodes for shielded metal arc welding. Miami: American Welding Society.

AWS A5.17. (2001) Specification for carbon steel electrodes and fluxes for Submerged Metal Arc Welding. Miami: American Welding Society.

Becerra, M. B., Zitzumbo, R., Domínguez J., García, J. L., Alonso, S. (2014). Use of the desirability function to optimize a vulcanized product. Rev. Téc. Ing. Univ. Zulia, 37(1), 85-94.

Cruz-Crespo, A., Quintana-Puchol, R., García, L. L., Perdomo, L., Jiménez, G., Gómez, C. R., Alguacil, F. J., Cores, A. (2005). Empleo de escorias de soldadura del sistema $\mathrm{MnO}-\mathrm{SiO}_{2}$ para la obtención de un nuevo fundente aglomerado aleado. Rev. Metal., 41(1), 3-11.

Cruz-Crespo, A., Perdomo-González, L., Fernández, R., Scotti, A. (2017). Composición química y microestructura del metal depositado con fundentes obtenidos con empleo de escorias del sistema $\mathrm{MnO}_{-} \mathrm{SiO}_{2-}$ CaO”. CentroAzucar, 44(3), 43-52.

Cruz-Crespo, A., Perdomo-González, L., Quintana-Pucho, R., Scotti, A. (2019). Fundente para recargue por soldadura con arco sumergido a partir de ferrocromo-manganeso y escoria de la reducción simultánea de cromita y pirolusita. Soldagem \& Inspeção, 24, 1-10.

Gulenç, B., Kahraman, N. (2003). Wear behaviour of bulldozer rollers welded using a submerged arc welding process. Materials and Design, 24, 537-542.

Jarre, C. M., Puig, R. A., Zamora-Ledezma C., Zamora-Ledezma, E. (2021). Caracterización preliminar de la ceniza de cáscara de arroz de la provincia Manabí, Ecuador, para su empleo en hormigones. Rev. Téc. Ing. Univ. Zulia, 44(1), 44-50.

John J. Coronado, J. J., Caicedo H. F., Gómez, A. L. (2009). The effects of welding processes on abrasive wear resistance for hardfacing deposits. Tribology International, 42, 745-749.

Kobe, S. (2007). Types of wear and suitable hardfacing filler metals. Kobelco Welding Today, 10(3), 3-7. 
Mendez, P. F., Barnes, N., Bell, K., Borle, S. D., Gajapathi, S. S., Guest, S. D., Izadi, H., Gol, A. K., Wood, G. (2014). Welding processes for wear resistant overlays. Journal of Manufacturing Processes, 16, 4-25.

Najarro-Quintero, R., Cruz-Crespo, A., Perdomo-González, L., Ramírez-Torres, J., Orbea-Jiménez M. (2018a). Empleo de escorias de horno cuchara y de cenizas de paja de arroz como componentes de un fundente para recargue por soldadura. Minería y Geología, 34(3), 331-344.

Najarro-Quintero, R., Cruz-Crespo, A., Perdomo-González, L., Ramírez-Torres, J., López R. (2018b). Potencialidades de las escorias de afino del acero en la obtención de un fundente para recargue por soldadura. CentroAzucar, 45(4), 32-40.

Perdomo-González, L., Quintana-Puchol, R., Cruz-Crespo, A., Gómez-Pérez, C. R. (2017). Obtaining of components of fluxes for submerged arc welding from the carbothermic reduction of chromite refractory. Rev. Téc. Ing. Univ. Zulia, 40(1), 42-51.

Potapov, N. N. (1989). Materiales para soldar: Gases protectores y fundentes. Moscú: Mashinoestroenie.

Quintana, R., Cruz, A., Perdomo, L., Castellanos, G., García, L. L., Formoso, A., Cores A. (2003). Eficiencia de la transferencia de elementos aleantes en fundentes durante el proceso de soldadura automática por arco sumergido. Rev. Metal., 39(1), 25-34.

Shan-Ping, L., Oh-Yang, K., Tae-Bum, K., Kwon-Hu, K. (2004). Microstructure and wear property of Fe-Mn$\mathrm{Cr}-\mathrm{Mo}-\mathrm{V}$ alloy cladding by submerged arc welding. Journal of Materials Processing Technology, 147, 191196.

Singh, K., Pandey, S. (2009). Recycling of slag to act as a flux in submerged arc welding. Resources, Conservation and Recycling, 53, 552-558.

Tusek, J., Suban, M. (2003). High-productivity multiple-wire submerged-arc welding and cladding with metalpowder addition. Journal of Materials Processing Technology, 133, 207-213. 


\section{REVISTA TECNICA}

DE LA FACULTAD DE INGENIERIA

UNIVERSIDAD DEL ZULIA

Vol. 44. №2, Mayo - Agosto, 2021

Esta revista fue editada en formato digital y publicada en Abril de 2021, por el Fondo Editorial Serbiluz, Universidad del Zulia. Maracaibo-Venezuela

www.luz.edu.ve

www.serbi.luz.edu.ve

www.produccioncientificaluz.org 